\title{
CFD investigation of respiratory flows in a space-filling pulmonary acinus model
}

\author{
J. Sznitman ${ }^{1}$, S. Schmuki ${ }^{1}$, R. Sutter ${ }^{1}$, A. Tsuda ${ }^{2}$ \& T. Rösgen ${ }^{1}$ \\ ${ }^{1}$ Institute of Fluid Dynamics, ETH Zurich, Switzerland \\ ${ }^{2}$ Physiology Program, Harvard School of Public Health, USA
}

\begin{abstract}
Due to the sub-millimetre dimensions and accessibility of the distal regions of the lung, respiratory flows in the pulmonary acinus are often difficult to assess. However, a realistic description of acinar flows is needed to understand aerosol transport and deposition for medical applications such as aerosol inhalation. In an effort to develop more realistic computational fluid dynamics (CFD) models of the pulmonary acinus, we have simulated convective flows under rhythmic breathing motion in a space-filling model of an acinar branching tree. Our model captures well the variety of 3D flow patterns present along the tree and confirms the existence of complex recirculating alveolar flows. Our results emphasize the role of the alveolar to ductal flow ratio in characterizing acinar flows. Lagrangian particle trajectories suggest that massless particles, not influenced by sedimentation or diffusion, stay principally confined within acinar ducts without entering into alveoli. The inherent modularity of the present model is well suited to create more complete geometries of acinar trees and investigate the influence of convective acinar flows on realistic sedimenting and/or diffusing particles.
\end{abstract}

Keywords: lung, respiration, pulmonary acinus, convective flows, CFD, Lagrangian particle tracking, aerosols, low-Reynolds number flows.

\section{Introduction}

The pulmonary acinus is characterized by the complex of alveoli arranged as a foam-like sleeve on the surface of peripheral airways [1]. Alveoli guarantee gas exchange with blood capillaries and are encountered past the terminal bronchioles distal to the trachea. While the first 14 proximal airway generations are conductive pipes distributing airflow, respiration is driven by pressure gradients between alveoli and the outside environment. These gradients are 
induced by lung parenchyma motion, which approximately satisfies geometric similarity [2]. Applying mass conservation results in a gradual deceleration of the airflow along the bronchial tree, such that acinar flows are effectively characterized by low Reynolds numbers (typically $R e<1$ ). However, such flows remain usually difficult to assess due to the sub-millimeter dimensions and the accessibility of the region.

Until present, insight into acinar flows has been brought by some experimental flow visualization studies [3, 4] and CFD models have made use of simple alveolated duct structures to investigate acinar flows [5-8]. In particular, low Reynolds alveolar flows can be extremely complex due to the unique alveolated duct structures and their time dependent motion. While convective acinar flows may not affect diffusive gas (oxygen) transport [9], alveolar flow patterns are, however, of significant relevance for aerosol kinematics and deposition of inhaled particles $[10,11]$.

Although there exists a history for the representation of alveoli figured as little spheres or hemispheres, it has been long recognized that alveoli are rather densely-packed hollow polyhedra [12]. Hence, in an effort to develop more realistic CFD models of the pulmonary acinus, we have developed a threedimensional acinar space-filling geometry to study respiratory convective flows.

\section{Numerical modelling}

\subsection{Space-filling geometry}

Our idealized model is based on the description of the acinar ventilatory unit by Fung [13], which assumes that all alveoli are equal and space-filling and ventilated as uniformly as possible. Of the existing geometries suggested for an alveolus, a suitable choice is the truncated octahedron (or 14-hedron). The main advantages of this alveolar geometry are that it is space-filling, enabling the assemblage of several polyhedra to form ducts without leaving any voids. It has the minimum surface area to volume ratio of all space-filling polyhedra, and the most common shapes for alveolar septa found in lung parenchyma are hexagons and rectangles [13].

Figure 1 shows how such polyhedra can be assembled into a space-filling acinar ductal tree. The structure of the tree is a 3D version of the schematic acinar model constructed by Fung [13], which bears resemblance with the description of Hansen et al. [14]. The acinar branching tree (fig. 1) is asymmetric and models a sub-region of an entire pulmonary acinus. Following the spacefilling assumption, all alveoli are identical and generation 8 consists of only one alveolus. Following Fung [13], the characteristic alveolar dimension is chosen to be $0.14 \mathrm{~mm}$. The entire geometry consists of 190 polyhedra for a total sub-acinar volume of $\sim 0.2 \mathrm{~mm}^{3}$ at $t=0(\sim 0.2 \%$ of the average volume of an entire acinus [1].

\subsection{Numerical methods}

Following self-similarity [2], any geometrical length scales with the $1 / 3$ power of the lung. Hence, our model is designed to breath in a sinusoidal manner, with a period $T$ such that: 


$$
L(t)=L_{0}\left[1+\frac{\beta}{2}+\frac{\beta}{2} \cdot \sin \left(2 \pi f t-\frac{\pi}{2}\right)\right],
$$

where $L_{0}$ is the length scale at $t=0$, and $f=1 / T$ is the breathing frequency. The volume excursion is given by $C=\left(V_{\max }-V_{\min }\right) / V_{\min }$, where $V_{\min }$ and $V_{\max }$ are the minimum and maximum volumes of the geometry. The length scale expansion factor is $\beta=(C+1)^{1 / 3}-1$. Breathing conditions model sedentary tidal breathing of an average adult with $T=3 \mathrm{~s}, C \approx 16.7 \%$, and $\beta \approx 5.3 \%$.

The equations of motion are governed by the unsteady, incompressible Navier-Stokes equations, expressed as:

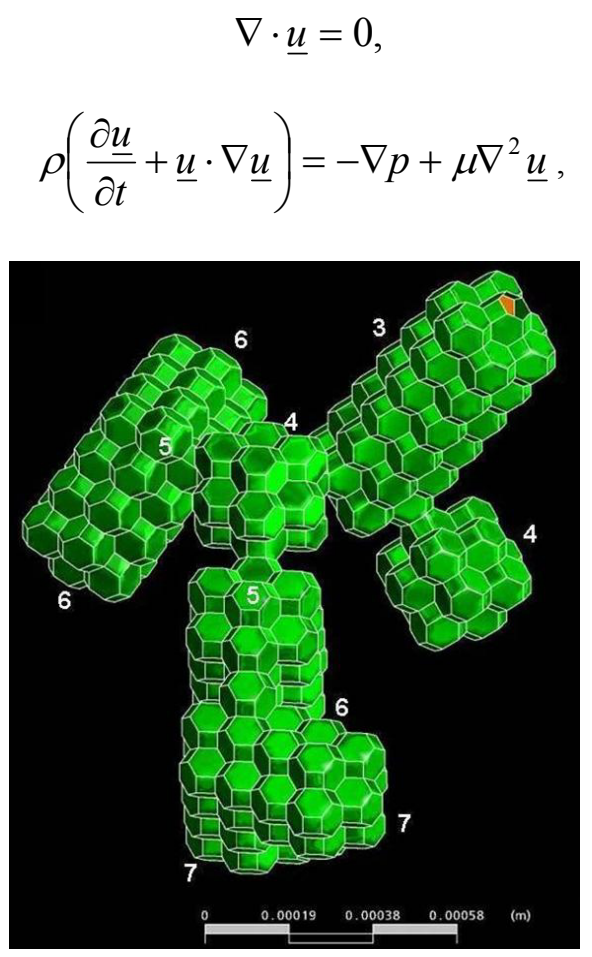

Figure 1: Acinar tree with generation numbering.

where $\underline{u}$ is the fluid velocity vector field, $p$ is the fluid pressure, $\rho$ its density and $\mu$ its dynamic viscosity. Laminar flow conditions are assumed [5] and external body forces are neglected. To obtain the time-dependent flow field, eqns. (2) and (3) are solved on a moving grid using a commercial finite-volume based program with fully implicit marching techniques (CFX-10, Ansys Inc.). Respiratory flows are generated by implementing the length scale displacement 
function (eqn. (1)). The no-slip condition was invoked along all acinar walls. The boundary at the entrance of the acinar tree was defined as a surface of constant pressure, arbitrarily set to 1 bar. Hydrodynamic similarity was achieved by matching Reynolds, $\operatorname{Re}=U D / v$, and Womersley numbers, $W_{O}=D \sqrt{f / v}$ to physiologically relevant values.

An unstructured mesh was generated for the geometry using a hybrid mesh. Simulations were performed to ensure that flow solutions were grid independent and converged, by performing a mesh refinement study. For this, we increased the density of volume elements by approximately $30 \%$ above the value that was eventually used ( $\sim 540^{\prime} 000$ elements). To achieve sufficiently accurate results such that changes in the resulting entrance flow $R e$ were within $<5 \%$ upon further time step refinement, a time step of $\Delta t=T / 200$ was eventually implemented [15]. Lagrangian trajectories of fluid (or massless) particles, $\underline{x}(t)$, were computed by integrating:

$$
\frac{d \underline{x}(t)}{d t}=\underline{u}(\underline{x}, t), \quad \underline{x}(t=0)=\underline{x}_{0}
$$

where $\underline{x}_{0}$ denotes an initial particle position.

\section{Results and discussion}

\subsection{Alveolar flow patterns}

Under rhythmical breathing, alveolar flows may substantially differ according to location along the airway tree (fig. 2). In the more proximal generations (gen. 3-5), flows are generally characterized by the existence of alveolar recirculation patterns. Flows in cavities recirculate slowly (compare alveolar and ductal velocities in fig. 3) and may exhibit the formation of open streamlines that spiral into singular points (fig. 2(a) and (b)). In contrast, in deeper generations (gen. 7, fig. 2(f)), or conversely past asymmetrical bifurcations leading to an airway end (gen. 4, fig. 2(e)), cavities are largely characterized by radial flows.

In proximal acinar generations, only a small portion of the fluid enters individual alveoli, while the bulk of the ductal flow is carried towards deeper generations to feed more distal alveoli. In turn, the relatively strong ductal flows in the proximal generations create a shear layer over the alveolar mouth openings. These in turn generate flow separation and ultimately a recirculation region within the cavities. Nevertheless, within each alveolus, the fluid near the walls travels along the walls and approaches the wall surface in the direction of wall motion due to breathing motion (fig. 3). Hence, alveolar flow patterns are determined by the interplay between shear flow over alveolar mouths, leading to recirculation, and radial streamlines induced by wall motion, as noted previously 
$[6-8,15]$. As one travels towards regions where the strength of the ductal flow decreases, recirculation regions disappear and fluid fills alveolar cavities in a radial fashion (fig. 2(f)).

\subsection{Ratio of alveolar to ductal flow rate}

Values of the alveolar to ductal flow ratio, $\dot{Q}_{a} / \dot{Q}_{d}$, capture well alveolar flow topologies (fig. 4). In particular, recirculation flows were abundantly present in proximal generations of the tree characterized by low values of $\dot{Q}_{a} / \dot{Q}_{d}$, which underlines that the ductal flow generates sufficient shear along the acinar mouths to create flow separation. Past a threshold value, $\dot{Q}_{a} / \dot{Q}_{d} \approx 0.02, \dot{Q}_{d}$ is too weak to induce recirculation. Our findings for $\dot{Q}_{a} / \dot{Q}_{d}$ are in good agreement with previous results for isolated alveolar cavities $[6,7,15]$. Note that from the definition of our space-filling model, $\dot{Q}_{a}=\dot{Q}_{d}$ at generation 8 , since the alveolus and duct are undistinguishable.

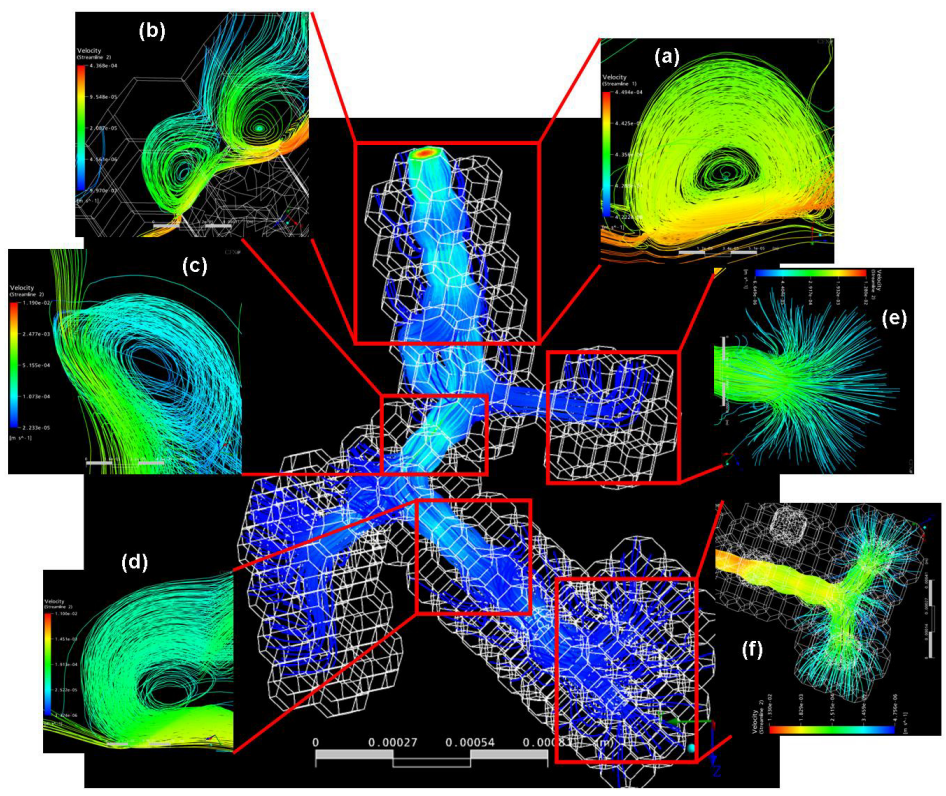

Figure 2: $\quad 3 \mathrm{D}$ ductal streamlines. Insets (a)-(f): examples of local alveolar flow fields. 


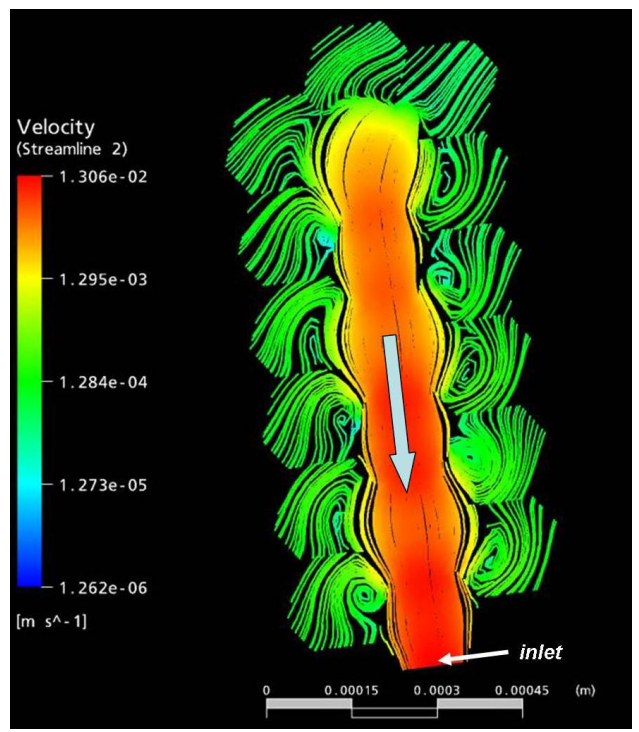

Figure 3: Cross-sectional velocity field during expiration $(t=0.8 \cdot T)$ in generation 3 .

Alveoli are by definition all identical. Hence, one might expect that alveolar flows be identical within a duct. However, looking at recirculating flow patterns (fig. 3) reveals in fact that neighboring alveolar flows differ slightly from one another. This specificity may arise from the fact that locally, each alveolus is oriented differently with respect to the streamwise ductal flow direction. In turn, the shear flow passing over an opening as well the fluid deflected into a cavity may be slightly affected by the alveolar orientation. Differences in flow patterns may also arise due to local changes in $\dot{Q}_{a} / \dot{Q}_{d}$. While $\dot{Q}_{a}$ is constant for any alveolus, $\dot{Q}_{d}$ decreases progressively with depth. Therefore, local changes in $\dot{Q}_{a} / \dot{Q}_{d}$ may account for such flow pattern differences.

\section{3 "Massless" particle trajectories}

Lagrangian particle tracks were examined to characterize the role of convective flows for aerosol transport. Particles were injected uniformly over the acinar inlet cross-section at a constant rate starting at $t=0$ (fig. 5). Resulting trajectories are strongly confined within alveolar ducts. Particles scarcely enter into alveolar cavities but rather, they follow the bulk of the ductal flow deep into the acinus. Within the end of an inspiration $(t=T / 2)$, particles have reached their most distal destination, effectively spreading along all branches. While particles follow streamlines, however, they cannot cross them (in contrast with sedimentation or diffusion mechanisms) and thus do not enter alveolar cavities. In the distal 
regions where $\dot{Q}_{a} / \dot{Q}_{d}$ (fig. 4) becomes relatively large as well as past asymmetrical airways leading to an airway end, convective velocities become so slow such that particles do not reach alveolar walls by the end of an inspiration. Hence, these particles stay within ducts and come short of entering alveolar cavities before the exhalation cycle begins and trajectories are reversed.

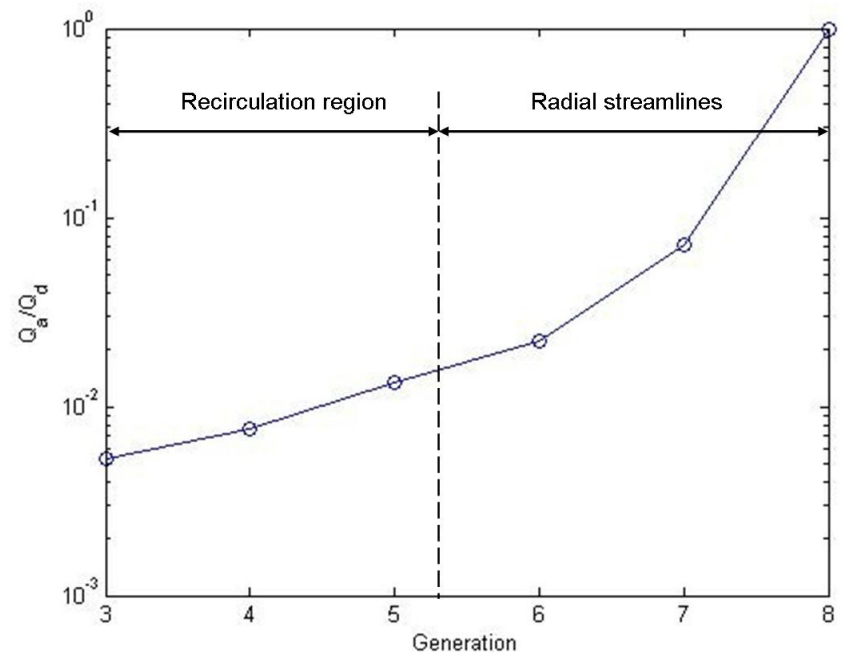

Figure 4: Ratio of alveolar to ductal flow rate.

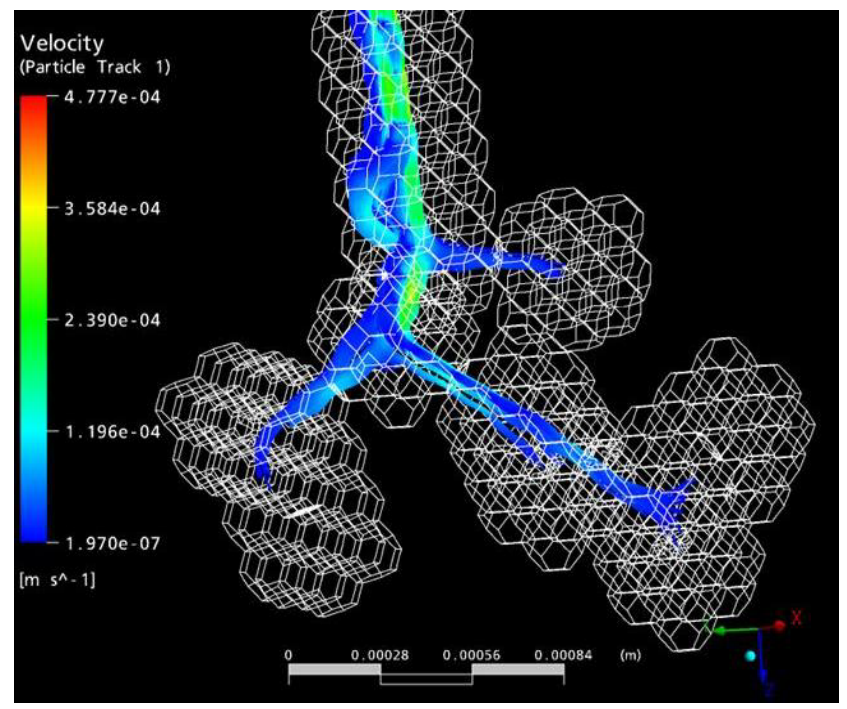

Figure 5: $\quad$ Massless particle trajectories at inspiration end $(t=T / 2)$. 
Results for massless particle trajectories seem to suggest that to reach bulk deposition of particles within alveolar cavities, additional transport mechanisms such as sedimentation or diffusion, depending on particle size, may play a considerable influence. Indeed, it has been previously shown that, depending on the size of aerosol particles (submicron or $>1 \mu \mathrm{m}$ ), it is rather the coupling of convection with either Brownian diffusion [10], or sedimentation under gravity [11], which leads to particle deposition at alveolar walls.

\section{Conclusions}

We simulated convective flows under rhythmic breathing motion in a spacefilling acinar tree model. Results capture well the existence of recirculating alveolar flows, similar to previous two- and three-dimensional studies $[6-8,15]$. Our results confirm again the importance of the alveolar to ductal flow ratio to characterize acinar flows. Lagrangian particle tracks suggest that particles starting at the model inlet are largely confined within acinar ducts. This result may suggest that while convection is an important mechanism for particle transport and mixing [16], it may not guarantee alone bulk particle deposition inside local alveolar cavities. By using the model's inherent modularity, we may modify the current geometry to construct new assemblages of more complete acinar networks. Such geometries may be used to investigate scenarios of collateral ventilation as well as pursue simulations of sedimenting and diffusing particles to examine the influence of acinar flows on realistic particle inhalation.

\section{References}

[1] Haefeli-Bleuer B, and Weibel ER, Morphometry of the human pulmonary acinus, Anat. Rec., 220, pp. 401-414, 1988.

[2] Ardila R, Horie T, and Hildebrandt J, Macroscopic isotropy of lung expansion, J. Appl. Physiol., 20, pp. 105-115, 1974

[3] Tippe A, and Tsuda A, Recirculating flow in an expanding alveolar model: experimental evidence of flow induced mixing of aerosols in the pulmonary acinus, J. Aerosol Sci., 31: 979-986, 1999.

[4] Tsuda A, Rogers RA, Hydon PE, and Butler JP, Chaotic mixing in the lung, Proc. Natl. Acad. Sci. USA, 99, pp. 10173-10178, 2002.

[5] Davidson MR, and Fitz-Gerald JM, Flow patterns in models of small airway units of the lung, J. Fluid Mech., 52: 161-177, 1972.

[6] Tsuda A, Henry FS, and Butler JP, Chaotic mixing of alveolated duct flow in rhythmically expanding pulmonary acinus, J. Appl. Physiol., 79: 10551063, 1995.

[7] Henry FS, Butler JP, and Tsuda A, Kinematically irreversible acinar flow: a departure from classical dispersive aerosol transport theories, J. Appl. Physiol., 92: 835-845, 2002.

[8] Haber S, Butler JP, Brenner H, Emanuel I, and Tsuda A, Shear flow over a self-similar expanding pulmonary alveolus during rhythmical breathing, J. Fluid Mech., 405: 243-268, 2000. 
[9] Sapoval B, Filoche M, and Weibel ER, Smaller is better - but not too small: a physical scale for the design of the mammalian pulmonary acinus, Proc. Natl. Acad. Sci. USA, 99: 10411-10416, 2002.

[10] Tsuda A, Butler JP, and Fredberg JJ, Effects of alveolated duct structure on aerosol kinetics. I. Diffusional deposition in the absence of gravity, $J$. Appl. Physiol., 76: 2497-2509, 1994.

[11] Tsuda A, Butler JP, and Fredberg JJ, Effects of alveolated duct structure on aerosol kinetics. II. Gravitational sedimentation and inertial impaction, J. Appl. Physiol., 76: 2510-2516, 1994.

[12] Mead J, Takishima T, and Leith D, Stress distribution in lungs: a model of pulmonary elasticity, J. Appl. Physiol., 28, pp. 596-608, 1970.

[13] Fung YC, A model of the lung structure and its validation, J. Appl. Physiol., 64, pp. 2132-2141, 1988.

[14] Hansen JE, Ampaya EP, Bryant GH, and Navin JJ, Branching pattern of airways and air spaces of a single human terminal bronchiole, J. Appl. Physiol., 38, pp. 983-989, 1975

[15] Sznitman J, Heimsch F, Heimsch T, Rusch D, and Rösgen T, Threedimensional convective alveolar flow induced by rhythmic breathing motion of the pulmonary acinus, ASME J. Biomech. Eng., in press.

[16] Heyder J, Blanchard JD, Feldman HA, and Brain JD, Convective mixing in human respiratory tract: estimates with aerosol boli, J. Appl. Physiol., 64, pp. 1273-1278, 1988. 